\title{
PLANTIO DE Ipomoea pes-caprae NAS DUNAS DA PRAIA BRAVA (ITAJAÍ, SC): COMPARAÇÃO DE DUAS TÉCNICAS
}

\author{
A. GOMES_NETO'; T. C. EMÍLIO; P. BOSA \& S. R. CUNHA² \\ Laboratório de Ecologia da Vegetação Costeira. CTTMar, Universidade do Vale do \\ Itajaí, Itajaí, SC. Rua Uruguai, nº 458, 88302-202, Brasil. \\ ${ }^{1}$ afraniogomesneto@yahoo.com.br; ${ }^{2}$ simone.cunha@univali.br
}

\begin{abstract}
RESUMO
Este trabalho teve como objetivo comparar, em campo, dois métodos para se propagar vegetativamente a planta Ipomoea pes-caprae: o plantio direto de estacas e o plantio de mudas enraizadas em viveiros antes do plantio em campo. Para tanto, foram coletados estolões provenientes de porções com entre-nós curtos, dos quais foram removidos brotos, folhas e raízes. Com estes estolões foram produzidos fragmentos de 20 e de $30 \mathrm{~cm}$, que foram enraizados em viveiro por 20 dias antes do plantio em campo (mudas; tratamento 1) ou plantados diretamente em campo (estacas; tratamento 2). No tratamento 1 as mudas tiveram suas folhas e brotos removidos antes do plantio em campo. As mudas e estacas de 20 e $30 \mathrm{~cm}$ foram identificadas, aleatorizadas e plantadas em uma área cercada das dunas (Praia Brava, Itajaí, SC) e sua sobrevivência foi acompanhada por um período de 2 meses. A maior mortalidade ocorreu nas primeiras três semanas após o plantio para todos os tratamentos, embora de maneira diferenciada para cada tamanho de fragmento. Os fragmentos de $30 \mathrm{~cm}$, tanto para as mudas como para as estacas, apresentaram valores de sobrevivência bem mais elevados do que os fragmentos de $20 \mathrm{~cm}$, tanto nas primeiras três semanas ( 83 e $76 \%$ de sobrevivência, para mudas e estacas, respectivamente), como ao final de 60 dias (74 e $62 \%$ de sobrevivência, para mudas e estacas, respectivamente). Apesar da elevada mortalidade observada para as mudas e estacas de $20 \mathrm{~cm}$ de comprimento nas primeiras 3 semanas do experimento (sobrevivência de 62 e $56 \%$, respectivamente), após este período houve uma redução na mortalidade, e o número de sobreviventes chegou, ao final de 60 dias, a 41 e $44 \%$ para mudas e estacas, respectivamente. Concluímos que para otimizar o plantio de Ipomoea pes-caprae nas dunas por meio de reprodução vegetativa, devem ser utilizados fragmentos de $30 \mathrm{~cm}$. A manutenção em viveiro aumenta a sobrevivência das plantas, e possivelmente terá forte influência do desempenho das plantas caso o plantio seja efetuado em períodos de baixa precipitação pluviométrica e sem regas freqüentes.
\end{abstract}

Palavras Chave: restauração de dunas; Ipomoea pes-caprae; propagação vegetativa

\section{PLANTING OF Ipomoea pes-caprae ON BRAVA BEACH DUNES (ITAJAÍ, SC): COMPARING TWO TECHNIQUES}

\begin{abstract}
This work aimed to compare survival of Ipomoea pes-caprae planted with two vegetative propagation methods: direct planting of cuttings and planting of cuttings rooted in a green house. Leaves and shoots were cut off of stolons with short distance among nodes. Stolon fragments of 20 and $30 \mathrm{~cm}$ were kept in a green house for rooting (rooted cuttings; treatment 1) or planted directly on the dune (non-rooted cuttings; treatment 2). Each cutting was marked and all were randomized and planted in a fenced area of Praia Brava (Itajaí, SC). Survival was monitored along two months. Higher mortality was observed in the first three weeks for all treatments, but it was different among treatments. The $30 \mathrm{~cm}$-cuttings of both treatments presented higher survival than
\end{abstract}




\begin{abstract}
$20 \mathrm{~cm}$-cuttings. It occurs in the first three weeks (survival of 83 and $76 \%$ to rooted cuttings and non-rooted cuttings, respectively), and also after 60 days (survival of 74 and $62 \%$ to rooted cuttings and non-rooted cuttings, respectively). Despite the high mortality of $20 \mathrm{~cm}$-cuttings in the first three weeks, survival after 60 days was still relatively high (survival of 41 and $44 \%$ to rooted cuttings and non-rooted cuttings, respectively). We concluded that $30 \mathrm{~cm}$-cuttings are better than $20 \mathrm{~cm}$-cutting, and rooted cuttings are better than non-rooted cuttings, especially when rainfall is low.
\end{abstract}

Key words: dunes restoration; Ipomoea pes-caprae; vegetative propagation.

\section{INTRODUÇÃO}

O fato das dunas costeiras desenvolverem um papel primordial na proteção e manutenção da linha de costa é consenso entre pesquisadores de todo o mundo. $\mathrm{O}$ ambiente de dunas é uma fonte essencial de sedimento para as praias durante eventos de alta energia de ondas, mantendo o dinâmico sistema praia/ duna e protegendo as áreas adjacentes contra processos erosivos, além de proporcionar habitats específicos para diversas espécies de plantas e animais (Seeliger, 1992; Hesp, 2001).

A cobertura vegetal desempenha uma função importantíssima para a manutenção de dunas costeiras vegetadas (Dewhurst, 2002). As plantas barram o sedimento que o vento sopra da praia e, conforme a vegetação nativa desenvolve suas raízes, rizomas e estolões, o pacote arenoso é fixado (Hesp, 2001). A perda da vegetação torna a praia e a duna mais suscetíveis à erosão causada por ventos e ondas e dificulta a recomposição destes ambientes após a passagem de ondas com alta energia (Dewhurst, 2002). De acordo com diversos autores o plantio de vegetação nativa deve ser a primeira providência a ser tomada para a reabilitação de dunas vegetadas, sendo importante a escolha de espécies pioneiras, que ocorram na região em questão e cujos métodos de propagação sejam conhecidos.

Ipomoea pes-caprae é uma planta pioneira, típica de dunas e com distribuição pantropical. Esta planta apresenta ramificações que tendem a fixar o pacote arenoso sob a área na qual se estabelece (Devall, 1992). É geralmente encontrada na porção frontal das dunas, sendo uma das espécies retentoras de sedimento mais comum no litoral centro-norte de Santa Catarina. Seu potencial para utilização em projetos de reabilitação de dunas já foi enfatizado por alguns autores (Hueck, 1955; Verhagen, 1998; Dewhurst, 2002; EPA, 2003). Entretanto, metodologias adequadas para sua propagação não foram descritas. Como Ipomoea pes-caprae se propaga tanto por sementes quanto por meios vegetativos, não há consenso sobre a principal forma de reprodução. Considera-se que características locais podem determinar o tipo predominante de propagação (Devall, 1992).

Considerando a importância de Ipomoea pes-caprae como fixadora de dunas e a sua abundância no litoral catarinense, esta espécie apresenta um grande potencial para utilização em projetos de recomposição da vegetação de dunas. Sendo assim, este trabalho teve como objetivo comparar, em campo, dois métodos para se propagar vegetativamente (a partir de estacas) a planta Ipomoea pes-caprae: o plantio direto de estacas e o plantio de mudas enraizadas em viveiros antes do plantio em campo.

\section{METODOLOGIA}

Estolões de Ipomoea pes-caprae foram coletados na Praia Brava, Itajaí, SC (Figura 1), mesmo local onde posteriormente foi realizado o plantio. Todas as folhas e brotos aéreos foram removidos do estolão para a produção das mudas e estacas. Raízes, se presentes, foram mantidas, mas podadas a um comprimen- 


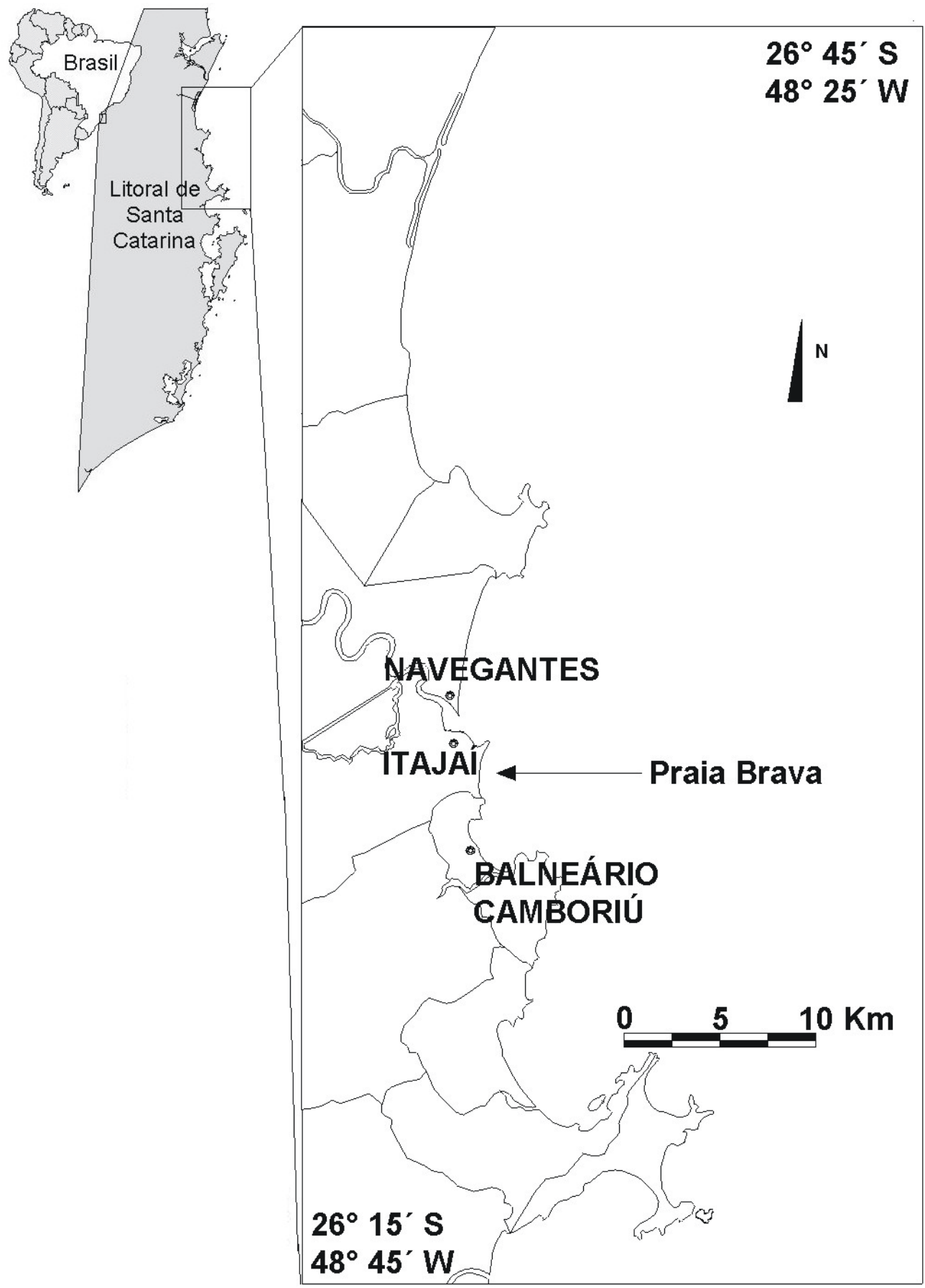

Figura 1 - Localização da área Praia Brava (Itajaí, SC), onde foi realizada a coleta e o plantio dos fragmentos de Ipomoea pes-caprae. 
to máximo de $5 \mathrm{~cm}$. Nos dois tratamentos testados foram utilizadas apenas porções nãoapicais de estolões com entre-nós curtos (estolões "secundários", com crescimento vertical, que partem do estolão principal, com crescimento horizontal), os quais foram considerados os mais adequados, conforme experimentos efetuados por Gomes-Neto (2004).

Para o plantio direto foram produzidos 27 fragmentos com $20 \mathrm{~cm}$ e 34 fragmentos com $30 \mathrm{~cm}$. Para o enraizamento das mudas em viveiro foram produzidos 42 fragmentos com 20 $\mathrm{cm}$ e 35 fragmentos com $30 \mathrm{~cm}$ de comprimento. Os fragmentos foram plantados em sacos plásticos contendo areia de praia peneirada e umedecida, sendo enterrados em torno de $10 \mathrm{~cm}$ de profundidade. Estas mudas foram mantidas por 20 dias em um viveiro coberto com tela com $50 \%$ de sombreamento. Antes do transplante para a duna, as folhas que se desenvolveram durante a permanência no viveiro foram removidas com um bisturi, para reduzir a transpiração da planta no campo, conforme determinado em experimentos anteriores (Gomes-Neto, 2004).

Os fragmentos plantados e enraizados em viveiro foram denominados de "mudas". Os fragmentos plantados diretamente em campo foram denominados de "estacas".

Todas as mudas e estacas foram plantadas no mesmo dia, em março de 2004. As mudas e estacas foram identificadas com etiquetas plásticas e aleatorizadas, sendo plantadas manualmente cerca de $35-50 \mathrm{~cm}$ de distância entre si, em uma área cercada para evitar o pisoteamento. Após o plantio foram distribuídos 160 litros de água doce e $30 \mathrm{~kg}$ de serragem sobre a área.

Periodicamente ao longo de dois meses, a sobrevivência foi acompanhada e todas as plantas tiveram seu número de folhas e comprimento da estaca medidos em campo. A sobrevivência foi representada como percentual do número inicial de plantas.

\section{RESULTADOS E DISCUSSÃO}

Não houve padrão definido na variação do número de folhas e do comprimento do estolão, impossibilitando sua análise. Portanto serão apresentados apenas os resultados de sobrevivência.

Os padrões de sobrevivência apresentados pelas mudas e estacas com mesmo comprimento foram bastante similares, conforme pode ser observado na figura 2. A maior mortalidade ocorreu nas primeiras três semanas após o plantio para todos os tratamentos, embora de maneira diferenciada para cada tamanho de fragmento.

Apesar da elevada mortalidade observada para as mudas e estacas de $20 \mathrm{~cm}$ de comprimento nas primeiras 3 semanas do experimento (sobrevivência de 62 e 56 \%, respectivamente), após este período houve uma redução na mortalidade, e o número de sobreviventes chegou, ao final de 60 dias, a 41 e $44 \%$ para mudas e estacas, respectivamente (Figura 2).

Os fragmentos de $30 \mathrm{~cm}$, tanto para as mudas como para as estacas (Figura 2), apresentaram valores de sobrevivência bem mais elevados do que os fragmentos de $20 \mathrm{~cm}$, tanto nas primeiras três semanas (83 e $76 \%$ de sobrevivência, para mudas e estacas, respectivamente), como ao final de 60 dias (74 e 62 $\%$ de sobrevivência, para mudas e estacas, respectivamente).

A maior mortalidade ocorrendo nas primeiras semanas pode ser considerada como conseqüência do estresse que as estacas e mudas sofrem ao serem plantadas na duna. A baixa capacidade de retenção de água pelo sedimento, aliada à ação do spray salino e às altas temperaturas que ocorrem nas dunas frontais, obrigam as plantas a despenderem parte de suas reservas energéticas para suportar estes fatores, diminuindo a quantidade de energia alocada para o crescimento e portanto, reduzindo as chances de sucesso do estabelecimento em campo. 
Apesar das mudas e das estacas terem apresentado valores elevados de sobrevivência, o processo de enraizamento prévio em viveiro favoreceu a sobrevivência das mudas, resultando numa diferença positiva de $12 \%$ de sobrevivência para as mudas e estacas de $30 \mathrm{~cm}$ e de $5 \%$ para mudas e estacas de $20 \mathrm{~cm}$. O melhor desempenho das mudas e estacas mais longas certamente está relacionado à maior quantidade de reserva acumulada no estolão, que favorece o estabelecimento da planta sob as condições adversas das dunas.

Os valores de sobrevivência observados neste estudo são bastante interessantes do ponto de vista de plantio de vegetação em dunas, sendo superiores aos observados por Reis \& Freitas (2002), que obtiveram $40 \%$ de sobrevivência ao utilizar propagação vegetativa de Ammophila arenaria. Estes valores estão também dentro da faixa considerada adequada para recuperação de vegetação de dunas por Verhagen (1998) e Dewhurst (2002), entre 50 e $80 \%$ de sobrevivência.

Com base nos resultados obtidos até o momento pode-se concluir que para otimizar o

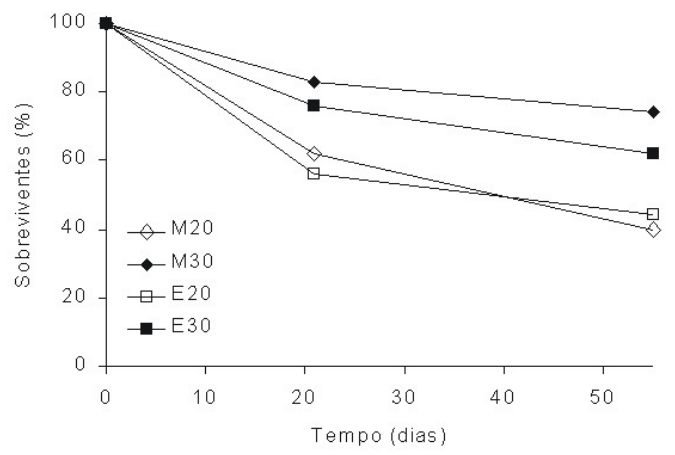

Figura 2 - Sobrevivência das mudas enraizadas em viveiro antes do plantio nas dunas e das estacas plantadas diretamente nas dunas. Códigos: M20: Mudas de 20 $\mathrm{cm}$ enraizadas em viveiro; M30: Mudas de $30 \mathrm{~cm}$ enraizadas em viveiro; E20: estacas de $20 \mathrm{~cm}$ plantadas diretamente nas dunas; E30: estacas de $30 \mathrm{~cm}$ plantadas diretamente nas dunas. plantio de Ipomoea pes-caprae nas dunas por meio de reprodução vegetativa, devem ser utilizados fragmentos de $30 \mathrm{~cm}$. Pode-se concluir também que a manutenção em viveiro aumenta a sobrevivência das plantas. A sobrevivência das mudas enraizadas em viveiro provavelmente será maior do que a de estacas plantadas diretamente, caso o plantio for efetuado em períodos de baixa precipitação pluviométrica e sem regas freqüentes. Entretanto experimentos complementares devem ser efetuados para verificar este caso. Outros pontos importantes que devem ser considerados em estudos posteriores são o plantio de mudas produzidas de sementes, para favorecer a diversidade genética das mudas, e o estudo de outras espécies típicas de dunas. Estratégias de recomposição da vegetação de dunas que incluam diversidade de espécies e mudas produzidas a partir de sementes e de fragmentos de rizomas/ estolões certamente serão mais bem sucedidas do que aquelas que utilizam reduzido número de espécies e apenas uma técnica de produção de mudas.

\section{REFERÊNCIAS BIBLIOGRÁFICAS}

Devall, M. S. 1992. The biological flora of coastal dunes and wetlands. 2. Ipomoea pescaprae (I.) Roth. Journal of Coastal Research. 8(2):442-456.

Dewhurst, D. (Comis.) 2002.Coastal Dunes: Dune protection and improvement manual for the Texas Gulf Coast. 2nd ed. Ed. Texas General Land Office. Disponível em: http:// www.glo.state.tx.us/coastal/dunemanual/. Acesso em: 14 de novembro de 2002.

EPA (Environmental Protection Agency - The State of Queensland). 2003. Goat's foot convolvulus (Ipomoea pes-caprae). Description of major dune plants IV-03 Coastal sand dunes: their vegetation and management 4. Disponível em: <http:// www.env.qld.gov.au/register/p00280aa.pdf >. Acesso em: 24 de julho de 2003. 
Hesp, P. A. 2001. Coastal Sand Dunes, Form and Function. Coastal Dune Vegetation Network Technical Bulletin no. 4.

Hueck, K. 1955. Plantas e Formação Organogênica das Dunas no Litoral Paulista. Parte 1: Contribuições para a pesquisa fitossociológica paulista 2. Secretaria da Agricultura do Estado de São Paulo.

Reis, C. S. \& H. Freitas, 2002. Rehabilitation of the Leirosa sand dunes. Littoral 2002, The Changing Coast. 381-384,
Seeliger, U. 1992.Coastal foredunes of southern Brazil: Physiography, habitats, and vegetation. p. 367-381. In: U. Seeliger, [Ed.], Coastal Plant Communities of Latin America. San Diego Academic Press.

Verhagen, H. J. 1998. Coastline Management, v.1. International Institute for Infrastructural, Hydraulic and Environmental Engineering. 